\title{
A systematic review and meta-analysis on the curative effects of cardiothoracic surgery for critical patients in the intensive care unit
}

\author{
Min Geng", Zhongping Li", Weidong Cui, Jiang Cheng, Liheng Li, Jinxia Li, Feng Li \\ Department of Critical Care, Jincheng People's Hospital (Jincheng Hospital Affiliated to Changzhi Medical College), Jincheng, China \\ Contributions: (I) Conception and design: M Geng, Z Li; (II) Administrative support: W Cui, J Cheng; (III) Provision of study materials or patients: \\ M Geng, Z Li, L Li, J Li, F Li; (IV) Collection and assembly of data: All authors; (V) Data analysis and interpretation: W Cui, J Cheng, Z Li, L Li, J \\ Li, F Li; (VI) Manuscript writing: All authors; (VII) Final approval of manuscript: All authors. \\ "These authors contributed equally to this work. \\ Correspondence to: Feng Li. Department of critical care, Jincheng People's Hospital (Jincheng Hospital Affiliated to Changzhi Medical College), No. \\ 456 Wenchang East Street, Jincheng 048000, China. Email: lifengjcsrmyy@163.com.
}

\begin{abstract}
Background The aim of this study was to explore the curative effects of cardiothoracic surgery (CTS) for critical patients in the intensive care unit (ICU) using meta-analysis.

Methods: Literature was searched using the following search terms: "cardiothoracic surgery", "intensive care unit", "critical patients", "post-operation", and "curative effects". Rev Man 5.3 was used for metaanalysis.

Results: A total of 15 randomized controlled trials (RCTs) were included, all of which had a low risk of bias, indicating medium and high quality. At 30 days after CTS, the number of patients with grade III and IV cardiac function was reduced by $76.84 \%$, and the overall heterogeneity test results revealed that $\mathrm{Tau}^{2}=0.09$, $\mathrm{Chi}^{2}=17.08, \mathrm{df}=5, \mathrm{I}^{2}=71 \%, \mathrm{P}=0.004<0.01, \mathrm{Z}=7.62, \mathrm{RR}=0.33$, and $95 \% \mathrm{CI}: 0.24$ to 0.43 . The incidence of adverse reactions was analyzed in 6 RCTs, and mainly manifested as improper anticoagulation thrombosis and bleeding. The overall heterogeneity test results revealed that $\mathrm{Chi}^{2}=1.07, \mathrm{df}=5, \mathrm{I}^{2}=0 \%, \mathrm{P}=0.96, \mathrm{Z}=4.93$, $\mathrm{OR}=0.46,95 \% \mathrm{CI}: 0.34$ to 0.63 , and $\mathrm{P}<0.01$. The 30 -day mortality rate was analyzed in $8 \mathrm{RCT}$. The overall analysis using the fixed effects model revealed that there was a notable difference between the experimental group and the baseline, with $\mathrm{Z}=10.11, \mathrm{OR}=0.12,95 \% \mathrm{CI}: 0.08$ to 0.18 , and $\mathrm{P}<0.01$.

Discussion: CTS can reduce the incidence of adverse events and the mortality rate of critical patients in the ICU, demonstrating high safety.
\end{abstract}

Keywords: Cardiothoracic surgery (CTS); intensive care unit; critical patients; curative effects

Submitted Jul 21, 2021. Accepted for publication Sep 09, 2021.

doi: 10.21037/apm-21-2224

View this article at: https://dx.doi.org/10.21037/apm-21-2224

\section{Introduction}

There are more than 1.6 million critical patients in the intensive care unit (ICU) undergoing cardiothoracic surgery (CTS) every year (1). After CTS, the most common postoperative complications are alveolar atrophy and thoracic cavity effusion (2), prolonging hospital stay and increasing costs. It is reported that the annual mortality rate of patients who have not undergone CTS is about $6 \%$, and the 5 -year mortality rate is as high as $60 \%$. Conservative treatment demonstrates limited efficacy, failing to prolong the survival time (3).

Critical patients in the ICU have declining systemic organ function and poor surgical tolerance. Among them, some patients suffer from chronic diseases, such as coronary heart disease, diabetes, and hypertension, and have engaged in long-term smoking, irregular physical exercise habits, 
and have low cardiorespiratory function capacity, increasing the risk of surgery (4). Additionally, during the process of CTS, cardiopulmonary function will further decline due to unavoidable anesthetics, surgical trauma stimulation, postoperative pain, and lung infection, severely damaging the body's immunity and negatively affecting the patient's rapid recovery (5). How to quickly recover from CTS is a challenge for both critical patients in the ICU and their doctors (6).

Cardiopulmonary exercise during the perioperative period enhances the blood supply of the heart and relieves the fatigue of the respiratory muscles so that the patient can inhale sufficient oxygen, further improving the body's tolerance, which is of great significance for the surgical rehabilitation of elderly patients (7). More importantly, postoperative cardiopulmonary exercise can significantly reduce the incidence of complications, and long-term and systematic cardiopulmonary exercise can improve the longterm quality of life of patients (8).

At present, there are an increasing number of patients undergoing CTS, but there are few randomized controlled trials (RCTs) and relevant studies to confirm the safety and effectiveness of CTS (9). The innovation of this study is that by collecting a large number of relevant literatures, concerning postoperative cardiac function classification, five-minute walking distance, echocardiography, adverse reactions, and the mortality rate of critical patients in the ICU undergoing CTS in order to deeply understand the postoperative curative effects and safety of CTS. This study is expected to provide a scientific basis for later clinical diagnosis and treatment. We present the following article in accordance with the PRISMA reporting checklist (available at https://dx.doi.org/10.21037/apm-21-2224).

\section{Methods}

\section{Literature review}

Chinese and English medical databases were searched from the establishment of the database to October 20, 2020, with "cardiothoracic surgery", "intensive care unit", "critical patients", "post-operation", and "curative effects" used as the search terms.

\section{Literature inclusion and exclusion criteria}

The inclusion criteria were as follows: (I) RCTs; (II) literature with critical patients in the ICU undergoing
CTS as subjects; and (III) literature containing indexes for evaluation outcomes.

The following studies were excluded: (I) literature with overlapping subjects; (II) literature not relevant to this study; and (III) literature with ambiguous results or missing indexes.

\section{Data extraction}

The following data were collated: (I) the title of the research; (II) the first author; (III) the name of the publication; (IV) year of publication; (V) the basic characteristics of the patients including age, gender, and number of cases; (VI) the intervention methods in the experimental group and the control group; and (VII) the evaluation indexes, including postoperative cardiac function, 5 -minute walking distance, echocardiography, incidence of adverse reactions, and mortality rate.

\section{Bias risk assessment and quality evaluation}

Two researchers conducted a bias risk assessment simultaneously. The risk of bias was evaluated by factoring in the 5 aspects of random allocation method, blinding method, allocation concealment, complete data, and research results.

The Newcastle-Ottawa Scale (NOS) was used to evaluate the quality of the literature according to 3 aspects (Table 1), namely patient selection, comparability of the study, and outcome. The score ranged from 0 to 9 . Studies with a score of 1 or less were considered low quality (high risk bias), studies with a score of $2-5$ were considered medium quality (unclear), and studies with a score of 6-9 were considered high-quality literature (low risk bias).

\section{Statistical analysis}

Stata SE13.0 was used for statistical analyses. Relative risk (RR), odds ratio (OR), mean difference (MD), and $95 \%$ confidence intervals (CI) were used to assess the postoperative cardiac function, 5 -minute walking distance, echocardiography, incidence of adverse reactions, and mortality rate of critical patients in the ICU undergoing CTS. RevMan 5.3 software was used to assess the risk of bias. When $\mathrm{P}>0.1$ and $\mathrm{I}^{2}<50 \%$, the fixed effects model (FEM) was used. When $\mathrm{P}<0.1$ and $\mathrm{I}^{2}>50 \%$, the random effects model (REM) was used. 
Table 1 The evaluation criteria of the Newcastle-Ottawa Scale (NOS)

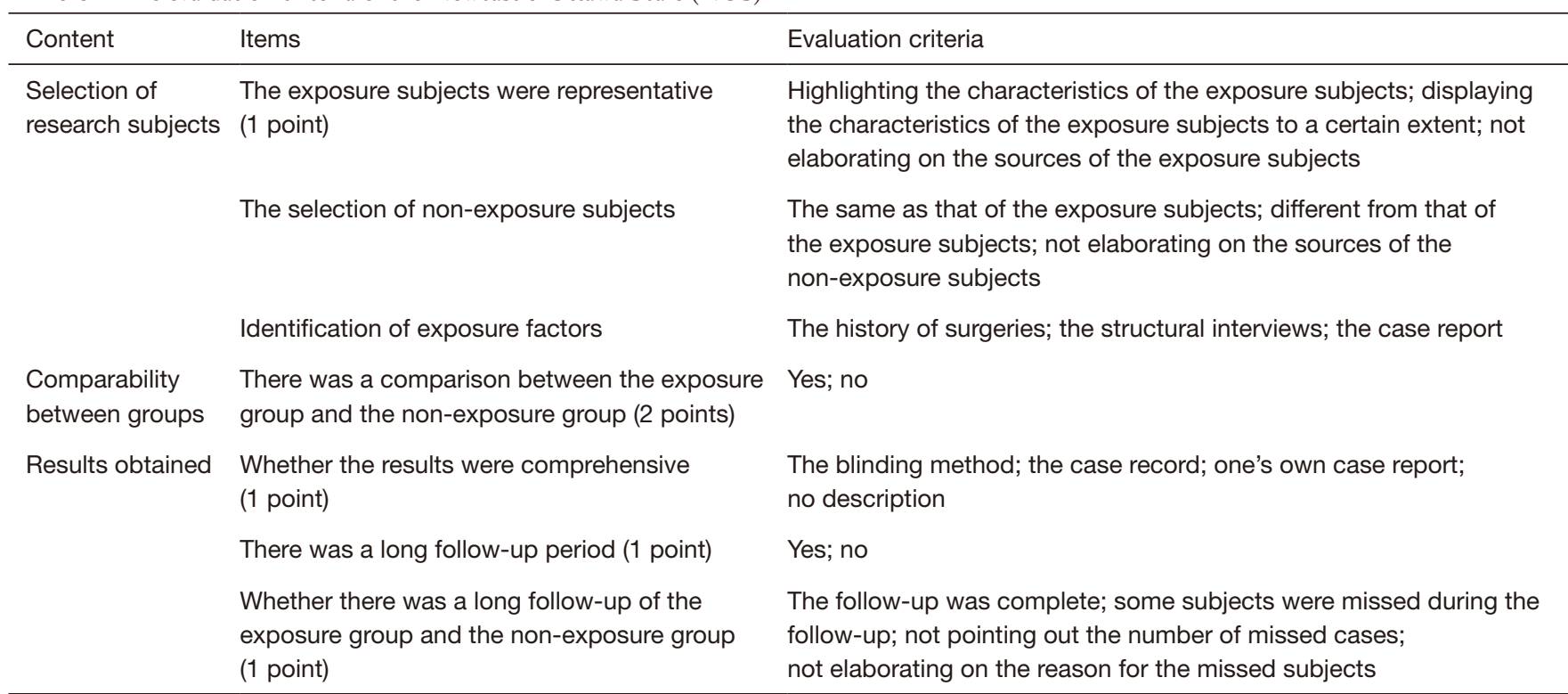

\section{Results}

\section{Basic information}

In this research, 315 literatures were obtained from the database and 309 literatures were obtained from the register. By reading the abstract and title of the articles, 131 literatures were repeatedly published, 109 were unqualified, and 58 were for other reasons, leaving 326 literatures. After reading the full text, 197 articles with repeated subjects were eliminated, leaving 129 articles. Eighty-one review reports were excluded, leaving 48 . Thirty-three articles that could not be extracted were excluded, and 15 articles were finally included in the study (Figure 1).

Figure 2 shows that 8 articles scored 5-9, 5 scored 2-4, and 2 scored 1 and below.

The 15 studies that met the inclusion criteria involved a total of 1,264 patients older than 18 years old, with sample sizes ranging from 34 to 96 . These studies were analyzed for the average age, number of cases, and intervention measures. The general characteristics of the research subjects are shown in Table 2.

\section{Risk of bias assessment}

Figures 3,4 are the results of the risk bias evaluation of studies plotted by Rev Man 5.3 software. Of the 15 RCTs, 3 (10-12) had the correct random allocation method and only $2(13,14)$ had allocation concealment. All studies adopted the blinding method.

\section{Postoperative cardiac function}

Postoperative cardiac function was analyzed in 6 RCTs, with a total of 812 cases involved. At 30 days after CTS, the number of patients with grade III and IV cardiac function was reduced by $76.84 \%$, and the overall heterogeneity test results revealed that $\mathrm{Tau}^{2}=0.09, \mathrm{Chi}^{2}=17.08, \mathrm{df}=5, \mathrm{I}^{2}=71 \%$, and $\mathrm{P}=0.004<0.01$. The research results by Paaske [2004] had the highest overlap with the combined results at $20.1 \%$, followed by the research results of Arcêncio [2008] (18.4\%). Additionally, the horizontal line was on the left side of the invalid vertical line at $95 \% \mathrm{CI}$ in all 6 studies. The overall analysis using the REM revealed that there was a notable difference between the experimental group and the baseline, with $Z=7.62, \mathrm{RR}=0.33$, and $95 \% \mathrm{CI}: 0.24$ to 0.43 (Figure 5).

\section{The five-minute walking distance}

The five-minute walking distance was analyzed in 4 RCTs, with a total of 546 cases involved. After CTS, the fiveminute walking distance increased by $75 \mathrm{~m}$, and the overall heterogeneity revealed that $\mathrm{Tau}^{2}=557.74, \mathrm{Chi}^{2}, 22.41, \mathrm{df}=3$, $\mathrm{I}^{2}=87 \%$, and $\mathrm{P}<0.01$. Additionally, the horizontal line was on the right side of the invalid vertical line at $95 \% \mathrm{CI}$ in all 4 studies. The overall analysis using the REM revealed that there was a notable difference between the experimental 
Identification of studies via databases and registers

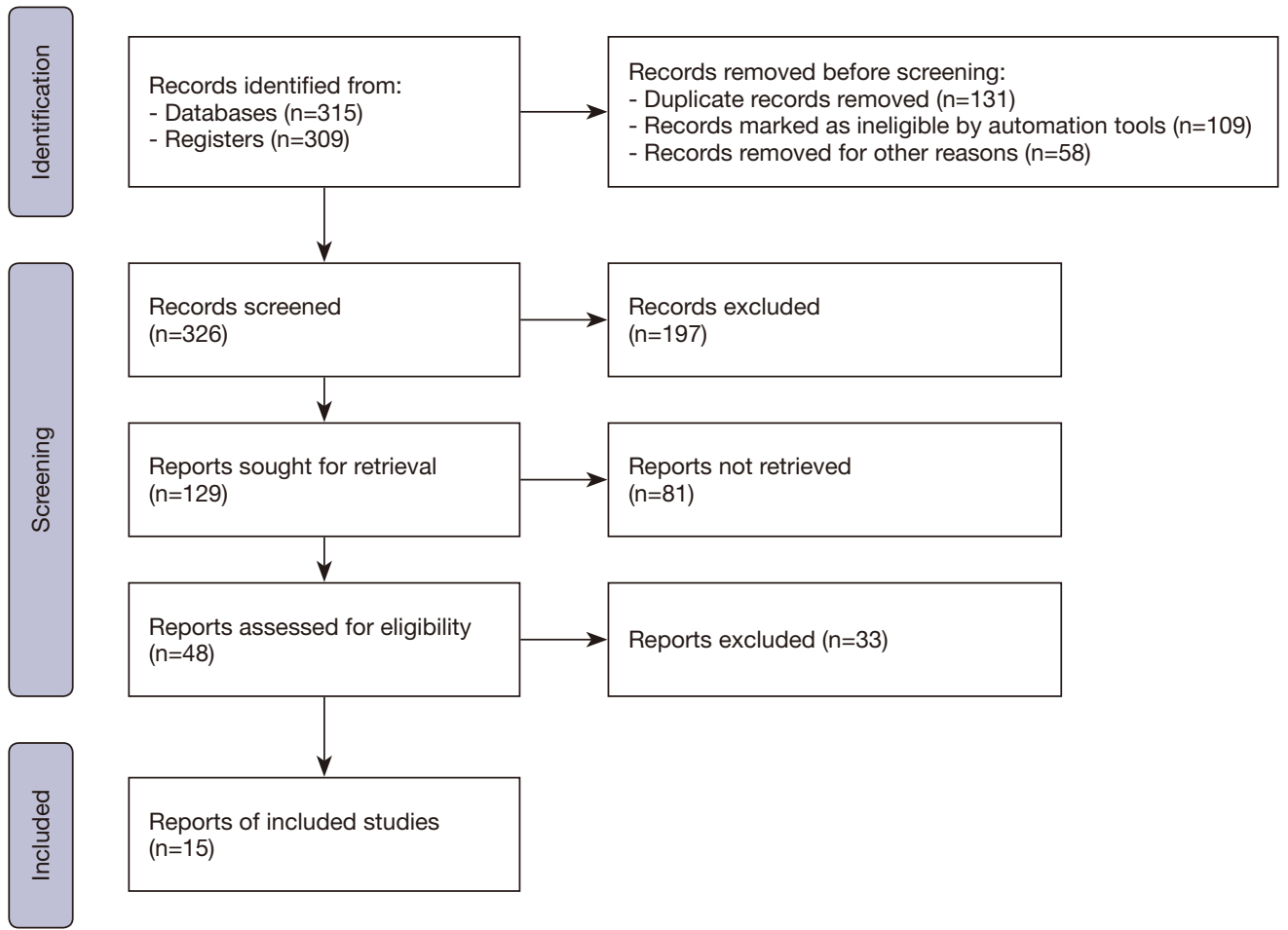

Figure 1 The literature search process.

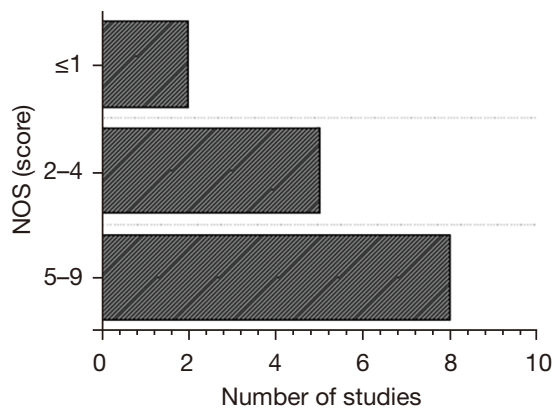

Figure 2 Quality classification results.

group and the baseline, with $\mathrm{Z}=11.56, \mathrm{MD}=147.34,95 \%$ CI: 122.39 to 172.35 , and $\mathrm{P}<0.01$ (Figure 6).

RevMan 5.3 was used to generate the five-minute walking distance funnel plot (Figure 7). It was noted that the circles in some studies were basically symmetrical along the midline, suggesting that the research accuracy was high and there was no bias in the publications.

\section{Echocardiography}

Echocardiography was analyzed in 6 RCTs, with a total of 862 cases involved. After CTS, the patients' left ventricular end-diastolic volume was reduced by $45.62 \mathrm{~mL}$ from baseline, and the overall heterogeneity test results revealed that $\mathrm{Tau}^{2}=237.40, \mathrm{Chi}^{2}=31.14, \mathrm{df}=5, \mathrm{I}^{2}=74 \%$, and $\mathrm{P}<0.01$. In 2 studies, the horizontal line was on the left side of the invalid vertical line at $95 \% \mathrm{CI}$. The overall analysis using the REM revealed that there was a notable difference between the experimental group and the baseline, with $\mathrm{Z}=8.58$, $\mathrm{MD}=-59.57,95 \% \mathrm{CI}:-73.17$ to -45.96 , and $\mathrm{P}<0.01$ (Figure 8 ).

RevMan 5.3 was used to generate the funnel plot of echocardiography (Figure 9). It was noted that the circles in some studies were basically symmetrical along the midline, suggesting that the research accuracy was high, and there was no publication bias.

After CTS, the patients' left ventricular end-diastolic diameter was reduced by $0.45 \mathrm{~cm}$ from baseline, and the overall heterogeneity test results revealed that $\mathrm{Tau}^{2}=0.05$, $\mathrm{Chi}^{2}=87.43, \mathrm{df}=5, \mathrm{I}^{2}=94 \%$, and $\mathrm{P}<0.01$. The horizontal line 
Table 2 The general characteristics of the research subjects

\begin{tabular}{|c|c|c|c|c|}
\hline Author & Year & Average age (year) & Number of cases & Cardiothoracic surgery \\
\hline Mollberg NM & 2013 & $46.29 \pm 11.35$ & 130 & Mitral valve replacement \\
\hline Stéphan F & 2016 & $31.53 \pm 8.36$ & 134 & Multi-center tricuspid valve surgery \\
\hline $\mathrm{Hu} \mathrm{A}$ & 2017 & $37.12 \pm 15.89$ & 118 & Single-center tricuspid valve replacement \\
\hline Arora KS & 2015 & $34.51 \pm 12.49$ & 72 & Mitral valve replacement \\
\hline Sade RM & 2008 & $46.59 \pm 15.48$ & 72 & Mitral valve clamp \\
\hline Kamarajah SK & 2019 & $36.59 \pm 12.49$ & 192 & Mitral valve replacement \\
\hline Hasegawa $T$ & 2014 & $36.54 \pm 14.45$ & 116 & Mitral valve clamp \\
\hline Arcêncio L & 2008 & $36.59 \pm 11.62$ & 184 & Single-center tricuspid valve replacement \\
\hline Okusanya O & 2020 & $46.74 \pm 12.41$ & 169 & Mitral valve replacement \\
\hline Vaporciyan AA & 2009 & $41.69 \pm 15.47$ & 148 & Mitral valve clamp \\
\hline Via MA & 2010 & $36.58 \pm 16.58$ & 171 & Mitral valve replacement \\
\hline
\end{tabular}

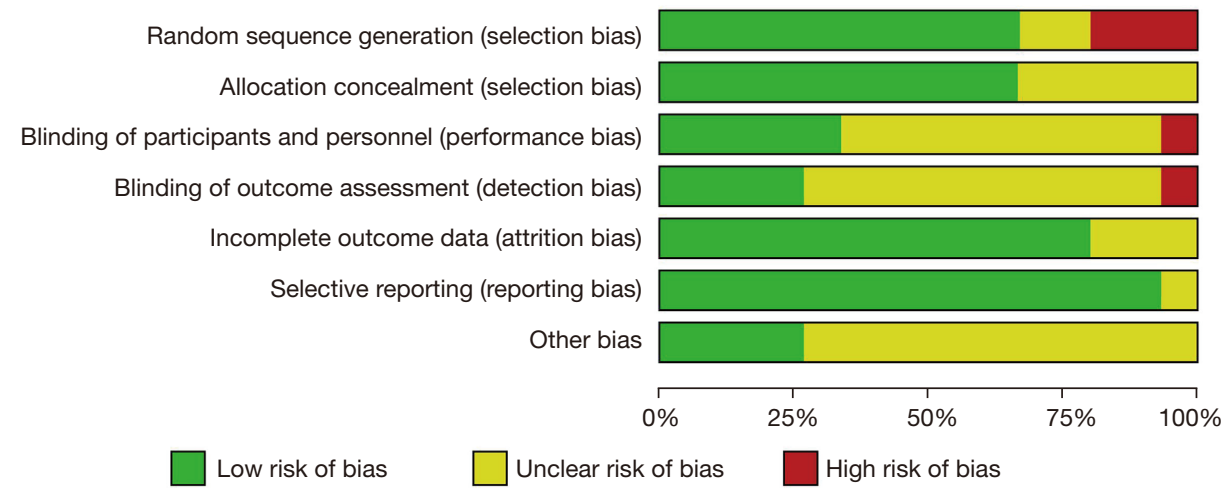

Figure 3 The risk of bias assessment.

was on the left side of the invalid vertical line at $95 \% \mathrm{CI}$ in 2 studies. The overall analysis using the REM revealed that there was a notable difference between the experimental group and the baseline, with $\mathrm{Z}=7.37, \mathrm{MD}=-0.66,95 \% \mathrm{CI}$ : -0.84 to -0.49 , and $\mathrm{P}<0.01$ (Figure 10).

\section{The incidence of adverse reactions}

The incidence of adverse reactions was analyzed in 6 RCTs, mainly manifesting as improper anticoagulation thrombosis and bleeding, with a total of 739 cases involved. The overall heterogeneity test results revealed that $\mathrm{Chi}^{2}=1.07, \mathrm{df}=5$, $\mathrm{I}^{2}=0 \%$, and $\mathrm{P}=0.96$. In some studies, the horizontal line crossed the invalid vertical line on the left at $95 \%$ CI. The overall analysis using the FEM revealed that there was a notable difference between the experimental group and the baseline, with $\mathrm{Z}=4.93$, OR $=0.46,95 \% \mathrm{CI}: 0.34$ to 0.63 , and $\mathrm{P}<0.01$ (Figure 11). 


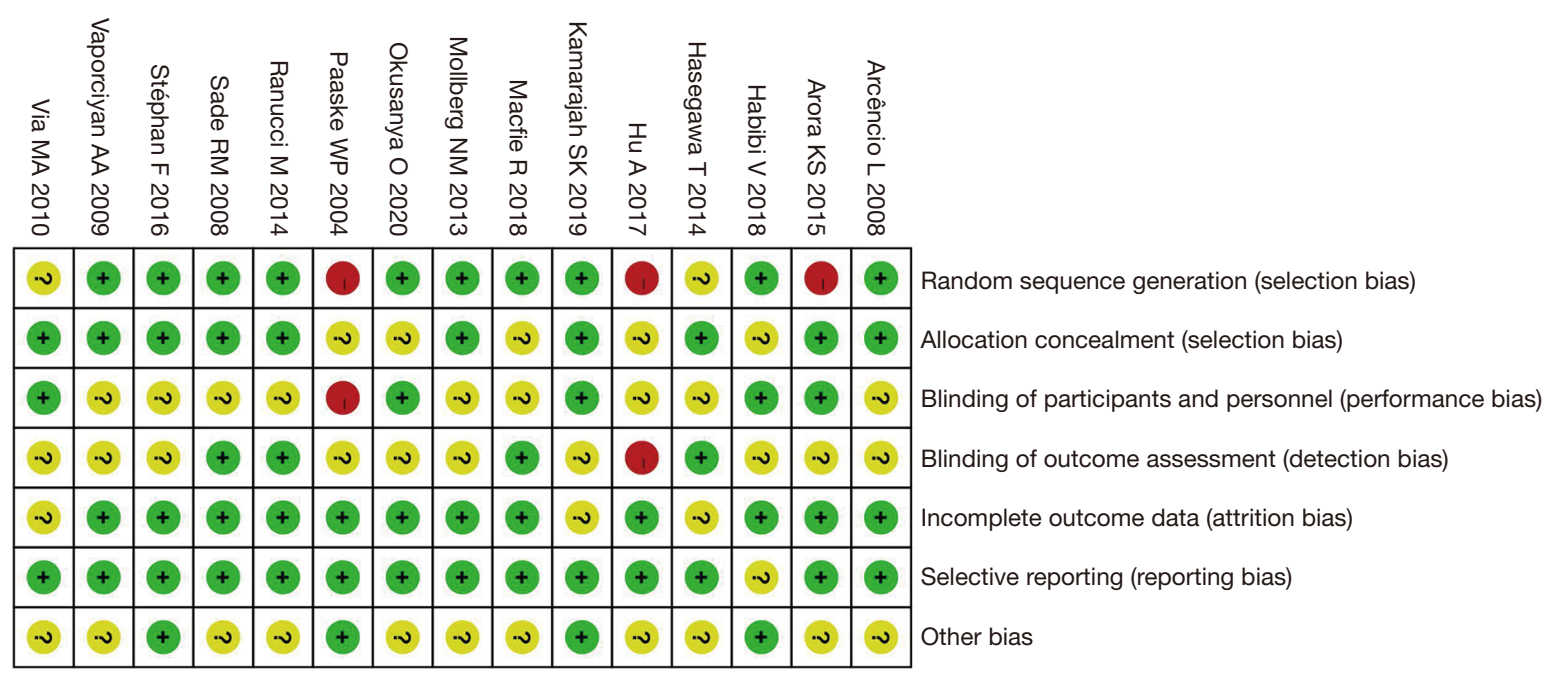

Figure 4 The multiple risk of bias evaluation results.

\begin{tabular}{|c|c|c|c|c|c|c|c|c|}
\hline \multirow[b]{2}{*}{ Study or Subgroup } & Experimental group & \multirow{2}{*}{\multicolumn{2}{|c|}{$\begin{array}{l}\text { Baseline } \\
\text { Events Total }\end{array}$}} & \multirow{2}{*}{ Weight } & \multirow{2}{*}{$\begin{array}{l}\text { Risk Ratio } \\
\mathrm{M}-\mathrm{H}, \text { Random, } 95 \% \mathrm{Cl}\end{array}$} & \multirow{2}{*}{\multicolumn{3}{|c|}{$\begin{array}{l}\text { Risk Ratio } \\
\text { M-H. Random. } 95 \% \mathrm{Cl}\end{array}$}} \\
\hline & Events Total & & & & & & & \\
\hline Arcêncio L2008 & 26 & 83 & 92 & $18.4 \%$ & $0.31[0.22,0.44]$ & $\rightarrow$ & & \\
\hline Hu A2017 & 15 & 54 & 59 & $15.5 \%$ & $0.28[0.18,0.43]$ & $\varpi$ & & \\
\hline Macfie R2018 & 39 & 32 & 38 & $12.1 \%$ & $0.27[0.15,0.49]$ & & & \\
\hline Mollberg NM2013 & 65 & 61 & 65 & $16.7 \%$ & $0.30[0.20,0.44]$ & $\rightarrow$ & & \\
\hline Okusanya O2020 & 21 & 79 & 84 & $17.3 \%$ & $0.26[0.18,0.38]$ & $\rightarrow$ & & \\
\hline Paaske WP2004 & 32 & 58 & 67 & $20.1 \%$ & $0.55[0.42,0.72]$ & $\rightarrow$ & & \\
\hline Total $(95 \% \mathrm{Cl})$ & 407 & & 405 & $100.0 \%$ & $0.33[0.24,0.43]$ & & & \\
\hline Total events & 121 & 367 & & & & & & \\
\hline Heterogeneity: $\mathrm{Tau}^{2}$ & .09; $\mathrm{Ch}^{2}=17.08, \mathrm{df}=5$ & $(P=0.00$ & 4); $; 1^{2}=$ & $71 \%$ & & 0.1 & 1 & 100 \\
\hline
\end{tabular}

Figure 5 The proportion of patients with grade III and IV cardiac function.

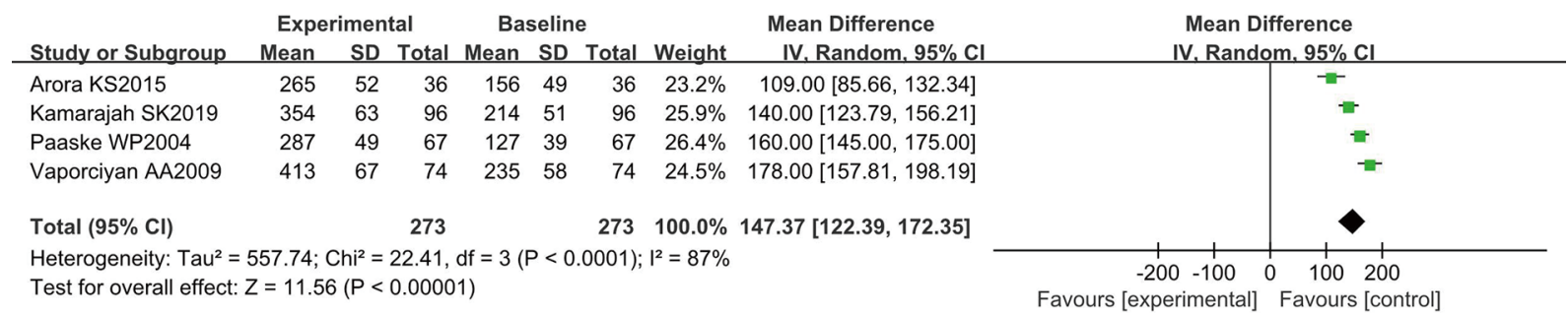

Figure 6 Forest plot for random effects model of five-minute walking distance.

\section{The 30-day mortality rate}

The 30-day mortality rate was analyzed in 8 RCTs, with a total of 1,073 cases involved. The horizontal line was on the left side of the invalid vertical line at $95 \%$ CI in 8 studies. The overall analysis using the FEM revealed that there was a notable difference between the experimental group and the baseline, with $\mathrm{Z}=10.11, \mathrm{OR}=0.12,95 \% \mathrm{CI}$ : 0.08 to 0.18 , and $\mathrm{P}<0.01$ (Figure 12).

RevMan 5.3 was used to generate a funnel plot of mortality (Figure 13). It was noted that the circles in some studies were basically symmetrical along the midline, 


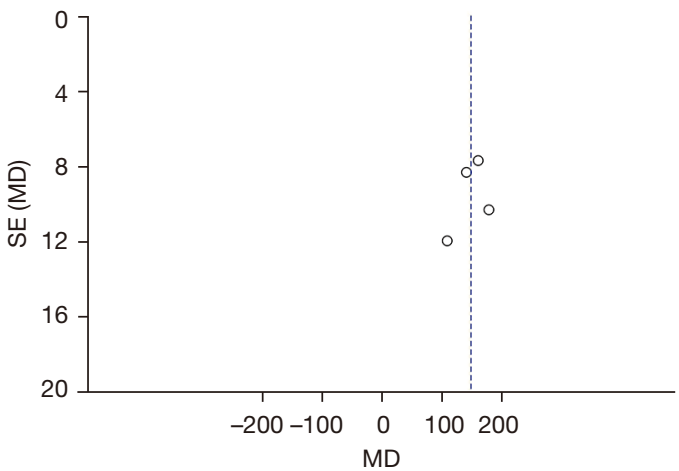

Figure 7 A funnel plot of a five-minute walk.

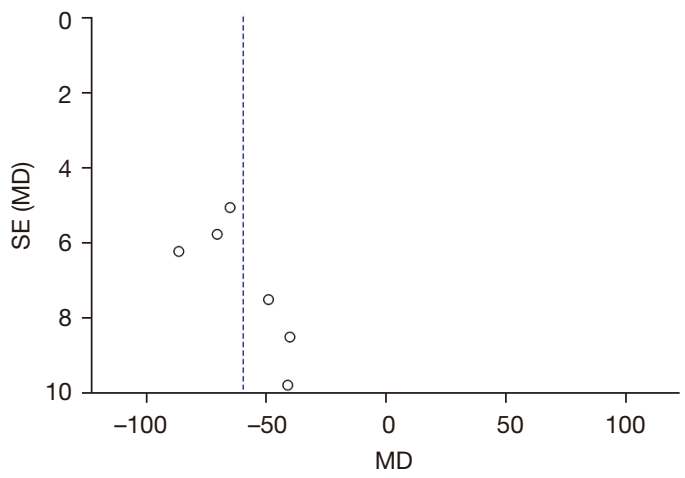

Figure 9 A funnel plot of echocardiography.

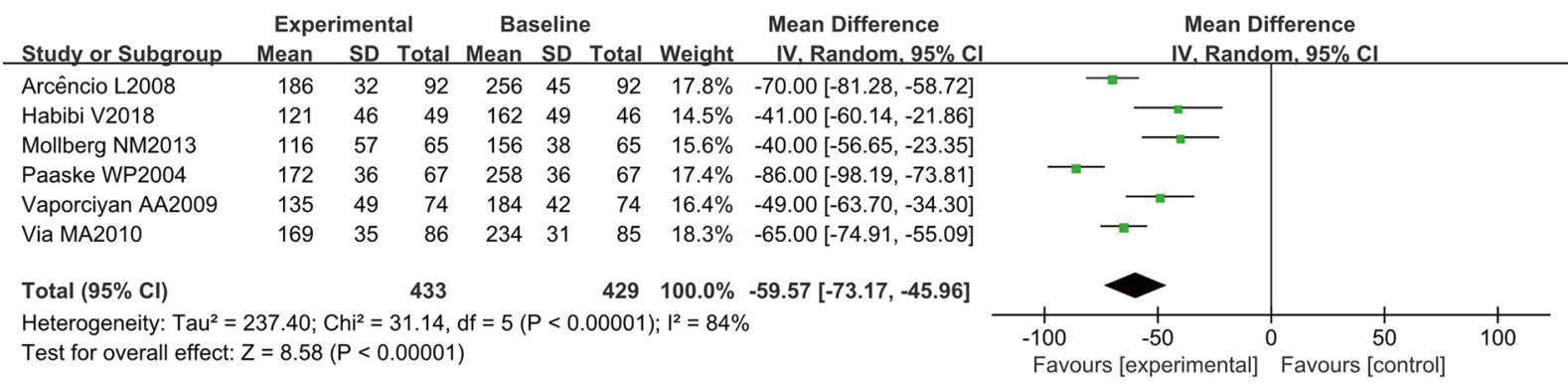

Figure 8 The left ventricular end-diastolic volume.

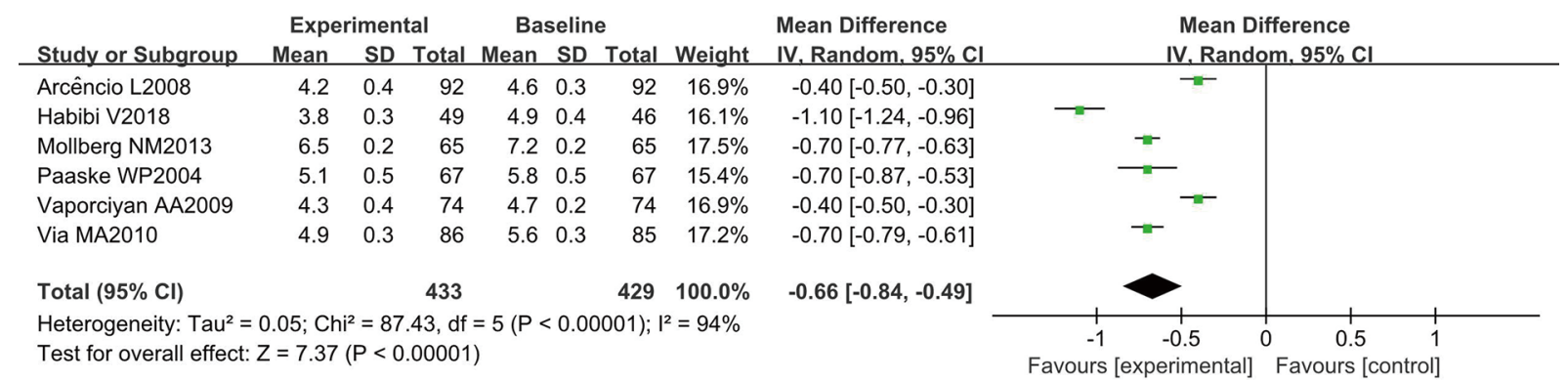

Figure 10 The left ventricular end-diastolic diameter.

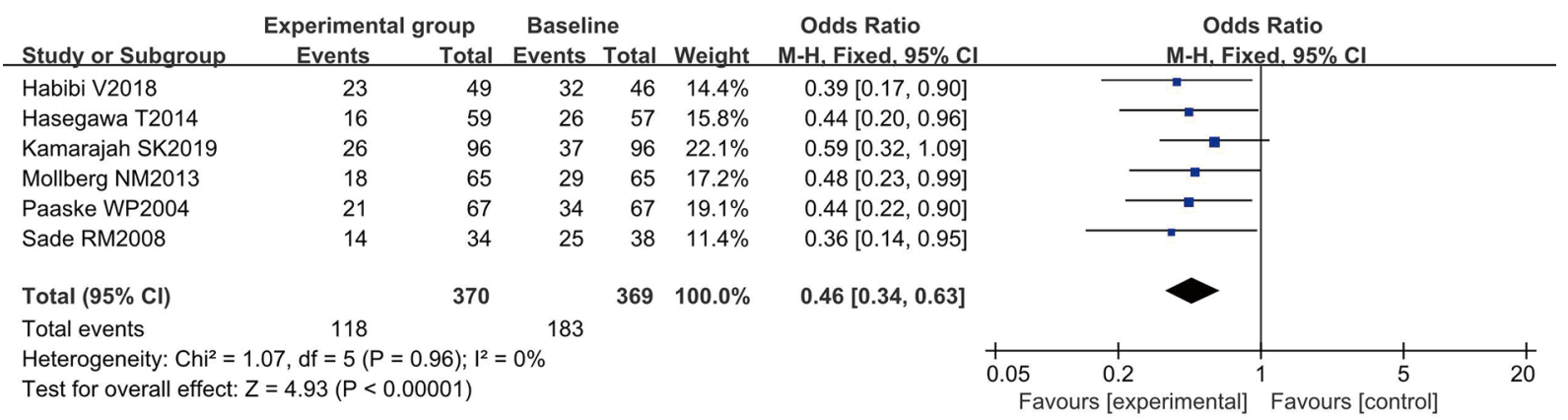

Figure 11 The incidence of adverse reactions. 


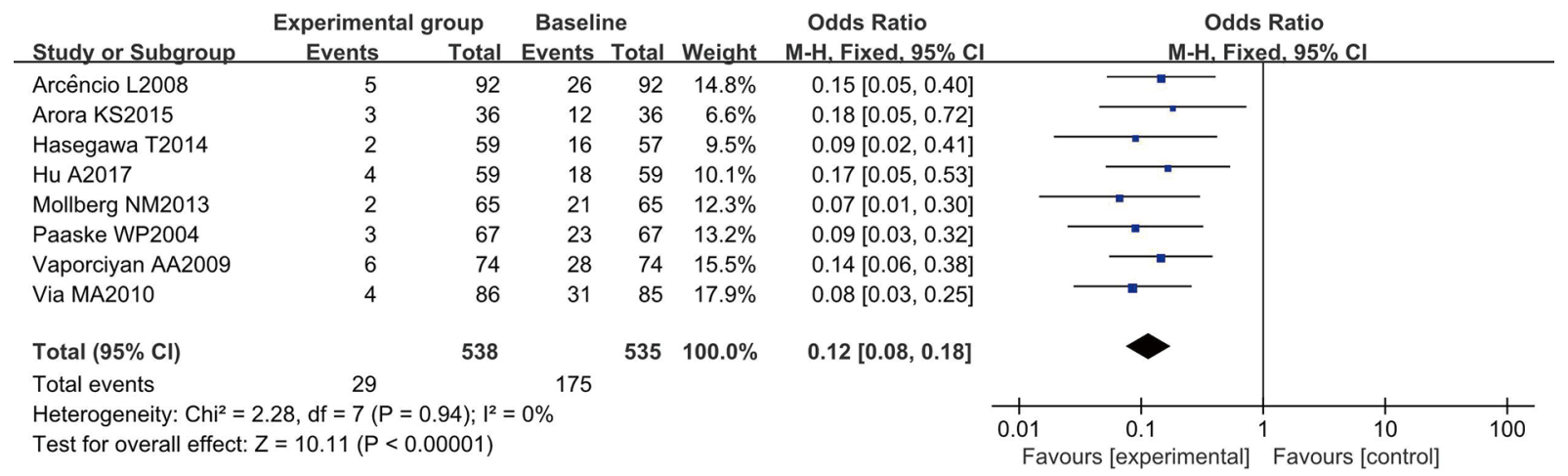

Figure 12 The mortality rate.

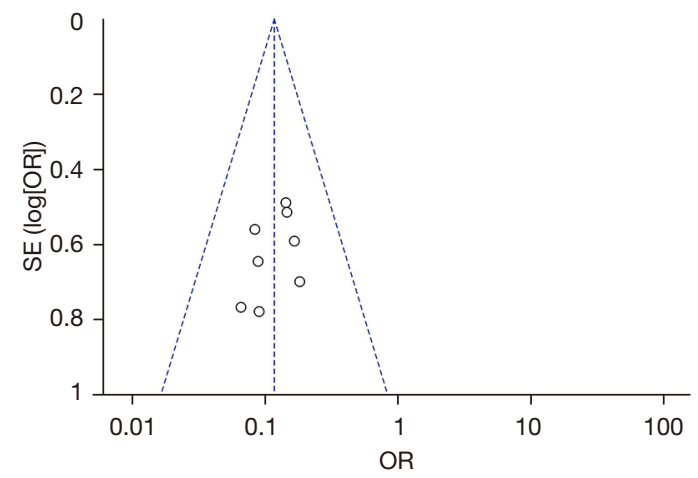

Figure 13 The mortality rate.

indicating that the research accuracy was high, and there was no bias in the publications.

\section{Discussion}

In this study, a meta-analysis was performed on the postoperative curative effects of CTS for critical patients in the ICU. The results found that the included literature demonstrated no bias (15).

At 30 days after CTS, the number of patients with grade III and IV cardiac function was reduced by $76.84 \%$, and the overall heterogeneity test results revealed that $\mathrm{Tau}^{2}=0.09$, $\mathrm{Chi}^{2}=17.08, \mathrm{df}=5, \mathrm{I}^{2}=71 \%$, and $\mathrm{P}=0.004<0.01$. The overall analysis using the REM revealed that there was a notable difference between the experimental group and the baseline, with $Z=7.62, R R=0.33$, and $95 \%$ CI: 0.24 to 0.43 . After CTS, there were $72.46 \%$ of critical patients with grade I and II cardiac function, lower than $87.49 \%$ reported by Chatterjee et al. [2019] (16).

The incidence of adverse reactions was analyzed in 6 RCTs, mainly manifesting as improper anticoagulation thrombosis and bleeding, with a total of 739 cases involved. The overall heterogeneity test results revealed that $\mathrm{Chi}^{2}=1.07$, $\mathrm{df}=5, \mathrm{I}^{2}=0 \%$, and $\mathrm{P}=0.96$. The overall analysis using the FEM revealed that there was a notable difference between the experimental group and the baseline, with $Z=4.93, O R=0.46$, 95\% CI: 0.34 to 0.63 , and $\mathrm{P}<0.01$, which was aligned with the research results of Shelley et al. [2019] (17).

The 30-day mortality rate was analyzed in 8 RCTs. The FEM revealed that there was a notable difference between the experimental group and the baseline, with $Z=10.11$, OR $=0.12,95 \%$ CI: 0.08 to 0.18 , and $\mathrm{P}<0.01$. The mortality rate in the study was $5.3 \%$, which was similar to $6.8 \%$ reported by Geoffrion et al. [2020] (18).

In the study, the 30-day mortality rate was $5.3 \%$, and 29 patients died 30 days after CTS. Adverse reactions included myocardial infarction, acute stroke, respiratory failure, bleeding, atrial fibrillation, infection, renal failure, and bleeding, with an incidence of about $31.89 \%$ (19). During the 1-year follow-up, 156 patients died, and the 1-year mortality rate was $28.9 \%$. The mortality rate and quality of life are important indicators to evaluate postoperative clinical efficacy (20). In the study, the different CTS methods had distinct impacts on the patients.

In this study, the included literature was classified based on the selection of subjects, the comparability between groups, and the results obtained using the NOS. There are some limitations of this study. The sample size was small, and the included literature was mostly of medium and high quality with high heterogeneity, and most of them were self-controlled trials. Hence, more clinical RCTs with larger samples sizes are needed. 


\section{Conclusions}

In this study, 15 publications were retrieved to explore the curative effects of CTS for critical patients in the ICU. The investigation confirmed that CTS can reduce the incidence of adverse events and the mortality rate of critical patients in the ICU, demonstrating high safety.

\section{Acknowledgments}

Funding: None.

\section{Footnote}

Reporting Checklist: The authors have completed the PRISMA reporting checklist. Available at https://dx.doi. org/10.21037/apm-21-2224

Conflicts of Interest: All authors have completed the ICMJE uniform disclosure form (available at https://dx.doi. org/10.21037/apm-21-2224). The authors have no conflicts of interest to declare.

Ethical Statement: The authors are accountable for all aspects of the work in ensuring that questions related to the accuracy or integrity of any part of the work are appropriately investigated and resolved.

Open Access Statement: This is an Open Access article distributed in accordance with the Creative Commons Attribution-NonCommercial-NoDerivs 4.0 International License (CC BY-NC-ND 4.0), which permits the noncommercial replication and distribution of the article with the strict proviso that no changes or edits are made and the original work is properly cited (including links to both the formal publication through the relevant DOI and the license). See: https://creativecommons.org/licenses/by-nc-nd/4.0/.

\section{References}

1. Ranucci M, Ballotta A, La Rovere MT, et al. Postoperative hypoxia and length of intensive care unit stay after cardiac surgery: the underweight paradox? PLoS One 2014;9:e93992.

2. Mollberg NM, Tabachnik D, Farjah F, et al. Utilization of cardiothoracic surgeons for operative penetrating thoracic trauma and its impact on clinical outcomes. Ann Thorac Surg 2013;96:445-50.
3. Stéphan F, Zarrouki Y, Mougeot C, et al. Non-Ventilator ICU-Acquired Pneumonia After Cardiothoracic Surgery: Accuracy of Diagnostic Tools and Outcomes. Respir Care 2016;61:324-32.

4. Hu A, Qiu Y, Zhang P, et al. Comparison of the effect of high versus low mean arterial pressure levels on clinical outcomes and complications in elderly patients during non-cardiothoracic surgery under general anesthesia: study protocol for a randomized controlled trial. Trials 2017;18:554.

5. Habibi V, Kiabi FH, Sharifi H. The Effect of Dexmedetomidine on the Acute Pain After Cardiothoracic Surgeries: A Systematic Review. Braz J Cardiovasc Surg 2018;33:404-17.

6. Arora KS, Khan N, Abboudi H, et al. Learning curves for cardiothoracic and vascular surgical procedures--a systematic review. Postgrad Med 2015;127:202-14.

7. Sade RM; American Association for Thoracic Surgery Ethics Committee; Society of Thoracic Surgeons Standards and Ethics Committee. Broadcast of surgical procedures as a teaching instrument in cardiothoracic surgery. J Thorac Cardiovasc Surg 2008;136:273-7.

8. Kamarajah SK, Bundred J, Weblin J, et al. Critical appraisal on the impact of preoperative rehabilitation and outcomes after major abdominal and cardiothoracic surgery: A systematic review and meta-analysis. Surgery 2020;167:540-9.

9. Hasegawa T, Oshima Y, Maruo A, et al. Pediatric cardiothoracic surgery in patients with unilateral pulmonary agenesis or aplasia. Ann Thorac Surg 2014;97:1652-8.

10. Paaske WP. The status of vascular surgery as an independent specialty in Europe: are the relationships with general and cardiothoracic surgery a problem? Vascular 2004;12:7-14.

11. Macfie R, Walcott-Sapp S, Watson J, et al. Association Between Simulation Curriculum and Learners' Confidence and Interest in Cardiothoracic Surgery. JAMA Surg 2018;153:968-9.

12. Arcêncio L, Souza MD, Bortolin BS, et al. Preand postoperative care in cardiothoracic surgery: a physiotherapeutic approach. Rev Bras Cir Cardiovasc 2008;23:400-10.

13. Okusanya O, Bartow A, Aranda-Michel E, et al. Resident perception of standardization and credentialing for high-risk bedside procedures in cardiothoracic surgery: Results from an institutional pilot study. J Card Surg 2020;35:2902-7. 
14. Vaporciyan AA, Reed CE, Erikson C, et al. Factors affecting interest in cardiothoracic surgery: Survey of North American general surgery residents. J Thorac Cardiovasc Surg 2009;137:1054-62.

15. Via MA, Scurlock C, Adams DH, et al. Impaired postoperative hyperglycemic stress response associated with increased mortality in patients in the cardiothoracic surgery intensive care unit. Endocr Pract 2010;16:798-804.

16. Chatterjee S, Shake JG, Arora RC, et al. Handoffs From the Operating Room to the Intensive Care Unit After Cardiothoracic Surgery: From The Society of Thoracic Surgeons Workforce on Critical Care. Ann Thorac Surg 2019;107:619-30.

17. Shelley BG, McCall PJ, Glass A, et al. Association between anaesthetic technique and unplanned admission to intensive care after thoracic lung resection surgery: the second Association of Cardiothoracic Anaesthesia and Critical Care (ACTACC) National Audit. Anaesthesia 2019;74:1121-9.

18. Geoffrion TR, Lynch IP, Hsu W, et al. An Implementation Science Approach to Handoff Redesign in a Cardiac Surgery Intensive Care Unit. Ann Thorac Surg 2020;109:1782-8.

19. Katz NM. The term "supportive care" is preferable to "palliative care" for consults in the cardiothoracic intensive care unit. J Thorac Cardiovasc Surg 2018;155:2030-1.

20. Keilin CA, Sandhu G, Matusko N, et al. Ten Years Into the Integrated Residency Era: A Pilot Study Shows Many Cardiothoracic Surgery Faculty Still Favor the Traditional Pathway. Semin Thorac Cardiovasc Surg 2020;32:756-62.

(English Language Editor: C. Betlazar-Maseh)
Cite this article as: Geng M, Li Z, Cui W, Cheng J, Li L, Li J, Li F. A systematic review and meta-analysis on the curative effects of cardiothoracic surgery for critical patients in the intensive care unit. Ann Palliat Med 2021;10(9):9879-9888. doi: 10.21037/apm-21-2224 